Rev. Elev. Méd. Vét. Pays trop., 1966, 19, 3 (323-330).

\title{
Leucocytozoon schoutedeni Rodhain, Pons, Vandendranden et Bequaert, 1913, chez la poule domestique, Gallus domesticus en Rhodésie.
}

\author{
par F. W. HUCHZERMEYER
}

\begin{abstract}
RÉSUMÉ
Leucocytozoon schoutedeni a été trouvé en plusieurs cas dans le noró de la Rhodésie chez la poule domestique exotique et indigène, Gollus domesticus. Une description du parasite est donnée ici. Le parasite est comparé en détail avec $L$. andrewsi et $L$. coulleryi et on suggère l'identité possible de $L$. andrewsi avec L. schoutedeni.
\end{abstract}

\section{INTRODUCTION}

Les membres du genre Leucocytozoon sont des protozoaires parasites des oiseaux. La schizogonie s'effectue dans les organes intérieurs. Les gamétocytes sont trouvés dans le sang circulant. Par la morphologie des gamétocytes et de leurs cellules-hôtes le genre peut être divisé en deux groupes, l'un ayant des formes allongées, en fuseau, l'autre des formes globuleuses. plus ou moins sphériques, Des représentants des deux groupes sont trouvés chez la poule domestique.

Berson (1964) ne reconnaît que L. sabrazesi comme seul membre valable du groupe allongé parasitant la poule domestique. L. caulleryi de forme sphérique - est caractérisé par le fait que les gamétocytes complètement matures se trouvent libres dans le sang (Dhanapala 1962 ; Seneviratna et al., 1963). RODHAIN et al. (1913) ont décrit un autre parasite de forme sphérique, $L$. schoutedeni, de la poule indigène dans ce qui était alors le Congo-belge. Dans cetfe espèce les gamétocytes maturés restent généralement intracellulaires. ATCHLEY (1951) rapportait la découverte d'un autre Leucocytozoon de forme sphérique chez la poule domestique en Caroline du Sud (U. S. A.) et le nommait $L$. andrewsi. COLES $(1939,1965)$ a vu un parasite ressemblant à $L$. coulleryi chez des poules de l'Afrique-du-Sud. Ce parasite paraissait dépourvu de pathogénicité.

\section{RÉSULTATS PERSONNELS EN RHODÉSIE}

a) Chez les poules domestiques exotiques.

Lors de la recherche de la cause de la mortalité dans un groupe de poules du type White Leghorn âgées de 6 mois dans la région de Karoi en mai 1965 cinq frottis de sang furent pris. Dans toutes ces préparations des gamétocytes sphériques de Leucocytozoon furent trouvés en petit nombre. Lors de la visite suivante en juin 1965, douze frottis de sang furent pris, et tous contenaient à nouveau un petit nombre de parasites. Un rapport entre le taux élevé de mortalité et le faible taux de parasitisme ne put être établi.

En décembre 1965 deux poules vivantes adultes de la race White Leghorn furent envoyées au Laboratoire de Recherches Vétérinaires d'Umvukwes. Toutes les deux furent trouvées infectées par le même Leucocytozoon. 


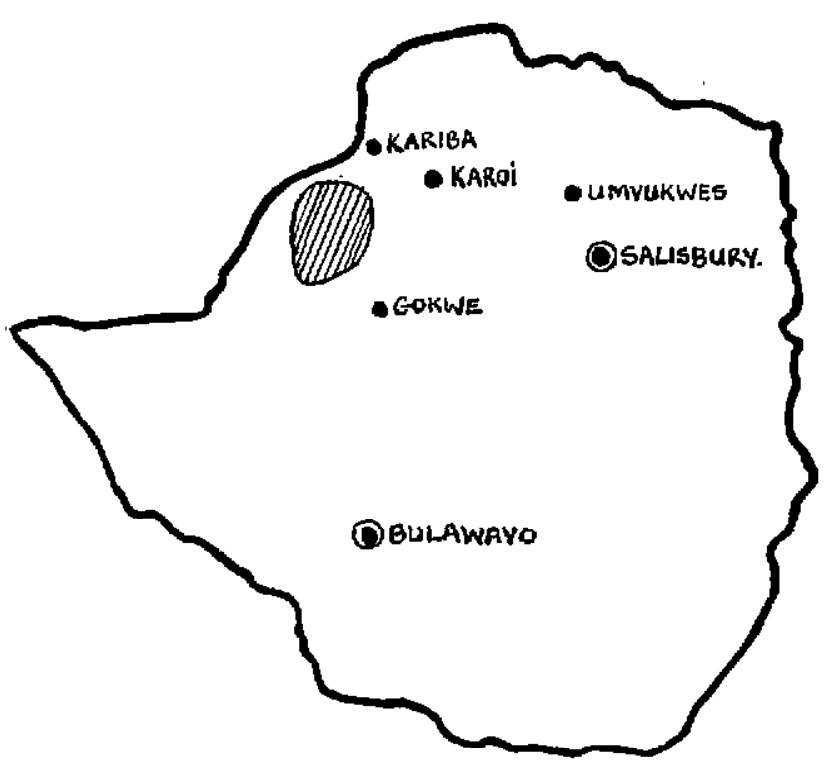

Carte l. - Rhodésie, La région striée indique les endroits où L. schoufedeni a été trouvé chez les poules domestiques indigènes.

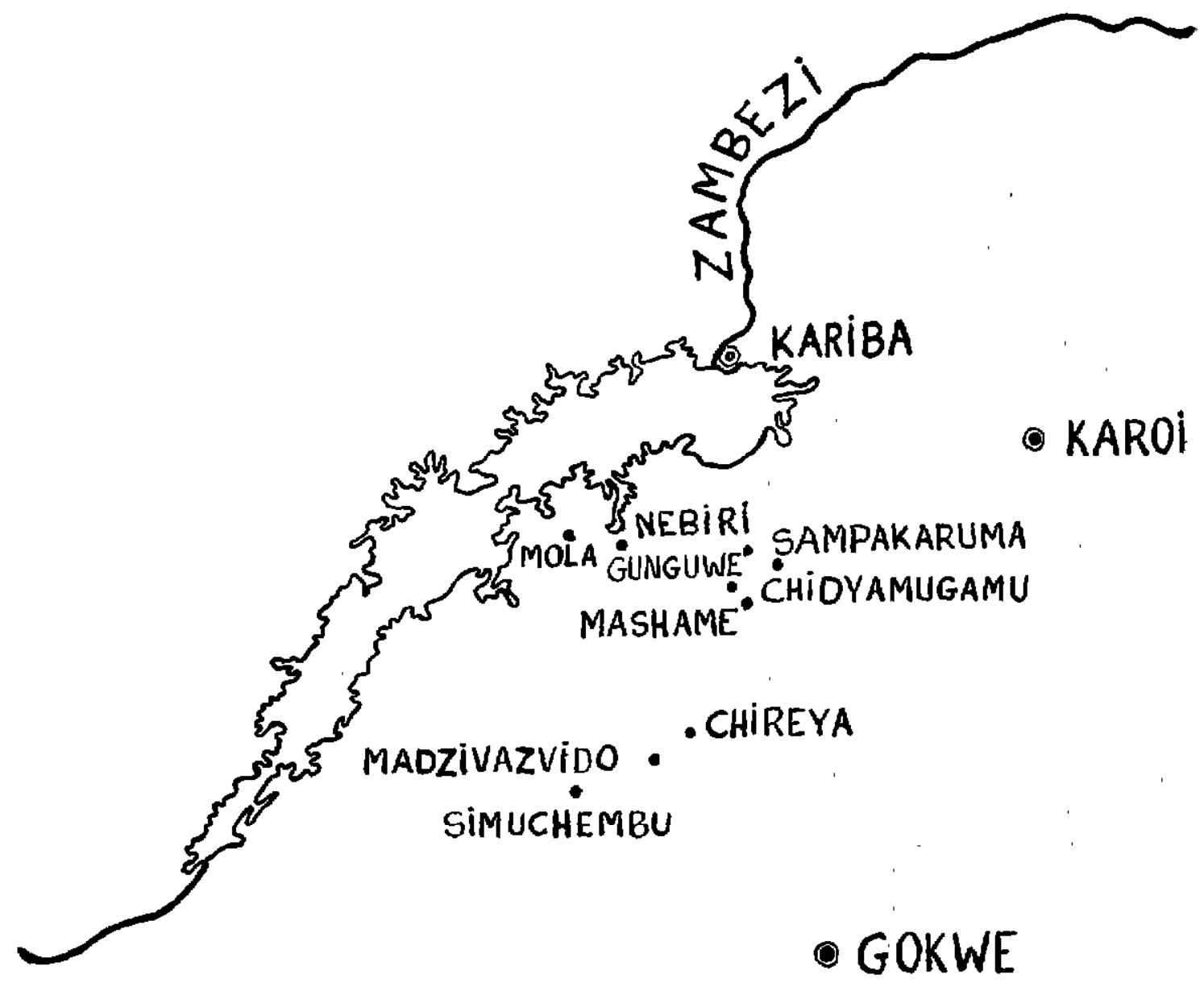

Carie II. - Région Gokwe Nord et Kariba de la Rhadésie. 
l'une montrant un nombre relativement élevé de gamétocytes.

b) Chez les poules domestiques indigènes.

Pendant une enquête sur les maladies des poules des Africains dans les districts du Nord de Gokwe et de Kariba 99 frottis de sang de poules indigènes adultes furent pris dans neuf localités différentes en mai 1965. Au moins un tiers de la surface totale de chaque frottis fut examiné sous fable grossissement ( 80 fois). Tout objet paraissant ressembler à un parasite fut alors examiné sous fort grossissement (immersion à l'huile, 800 fois). Les mêmes gamétocytes sphériques de Leucocytozoon furent trouvés dans 58 des frottis. Toutes les poules dont les frottis furent pris paraissaient être en bonne santé avec une seule exception. Cette poule présentait une anémie sévère et on découvrit plus tard qu'elle avait souffert d'Aegyptianellose. Aucune des neuf localités n'était exempte d'infection à Leucocytozoon. Chez quelques poules le taux de parasitémie était extrêmement bas (1 à 2 parasites par frottis), chez d'autres relativement élevé (plus de 30 parasites par frottis).

La distribution des parasites sur les frottis pris dans les localités différentes s'établissait comme suit :

Simuchembu ............ 9 frottis positifs sur un total de 23

Madzivazvido ............. 1 sur 10

Chireya ............... 8 sur 12

Mashame ................ 13 sur 13

Sampakaruma ........... 3 sur 7

Chidyamugamu ............ 10 sur 10

Nebiri................... 6 sur 7

Mola .................. 1 sur 7

Gunguwe $\ldots \ldots \ldots \ldots \ldots \ldots .7$ sur 10

\section{c) Description du parasite.}

Les schémas de 30 gamétocytes (25 femelles et 5 mâles) furent pris à la chambre claire. Ces schémas furent aussi la base des mesures et de la description données plus bas. Le noyau de la cellule-hôte se colore au Giemsa aussi intensément et de la même couleur que le noyau d'un érythrocyte. II est étiré aufour du parasite et embrasse un à deux tiers de la circonférence du parasite. Une marge de cytoplasme rosé jaune très pâle est habituellement située autour du parasite en face du noyau. Le macrogamétocyte prend une teinte bleu foncé et son cytoplasme contient souvent un nombre de granules violet foncé et de petites structures vacuolaires. Le petif noyau d'habitude de forme allongée, irrégulière est toujours pourvu d'un petit caryosome distinct. L'examen de 25 macrogamétocytes a montré que le caryosome était situé à l'extrémité du noyau dans 12 cas, en position marginale autre que l'extrémité dans 11 cas et à l'intérieur du noyau en deux cas.

Le microgamétocyje prend une teinte rose, Granules et vacuoles se trouvent beaucoup moins fréquemment que dans le macrogamétocyte. Le noyau est relativement large. On ne voit d'ordinaire pas de caryosome.

Les mesures données plus bas sont celles données par 25 macrogamétocytes et de 5 microgamétocytes respectivement.

macrogamétocyte cellule-hôte diamètre moyen

$15,1 \mu$ (de $12,5 \mu$ d̀ $17,5 \mu$ )

macrogamétocyte diamètre moyen $11.8 \mu$

(de $9.9 \mu$ à $13,3 \mu$ )

macrogamétocyte noyau longueur moyenne $4,7 \mu \quad$ (de $3,5 \mu$ à $6 \mu$ )

microgamétocyte cellule-hôte diamètre moyen $14,6 \mu$ (de 13,1 $\mu$ d̀ 16.4 $\mu$ )

microgamétocyte diamètre moyen $11,3 \mu$

(de $10,4 \mu$ à $11,9 \mu$ )

microgamétocyte noyau longueur moyenne $9,3 \mu$ (de $8 \mu$ à $10 \mu$ )

\section{DISCUSSION}

Au tableau I on trouvera un résumé des descriptions de L. schoutendeni, L. andrexsi, L. caulleryi, et du Leucocytozoon de la poule, trouvés en Rhodésie.

L. coulleryi diffère surtout par l'absence de la cellule-hôte. Le macrogamétocyte n'a pas de granulation son noyau est sphérique. Les gamétocytes des deux sexes paraissent être plus petits que les mesures des trois autres descriptions. Les quteurs semblent différer d'avis en ce qui concerne le pouvoir pathogène de ce parasite. GRIFFITH (1963) souligne particulièrement la gravité des crises en Extrême-Orient. 


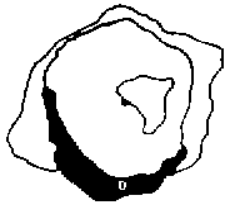

1
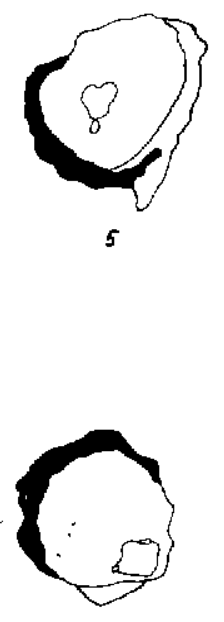

9

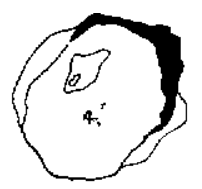

B

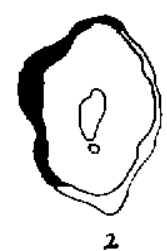

2
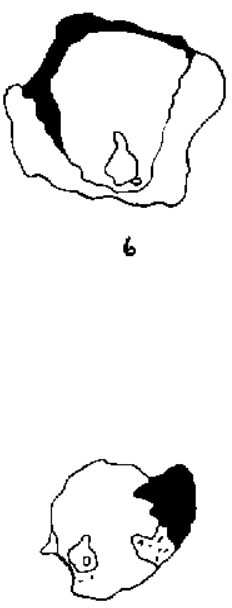

10

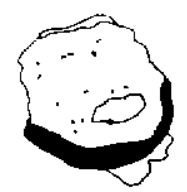

14
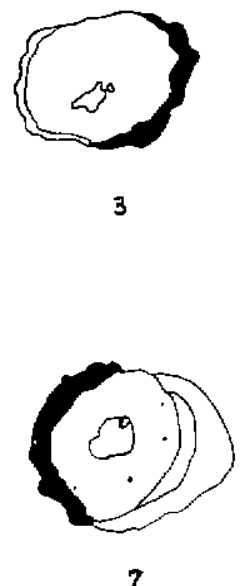

7
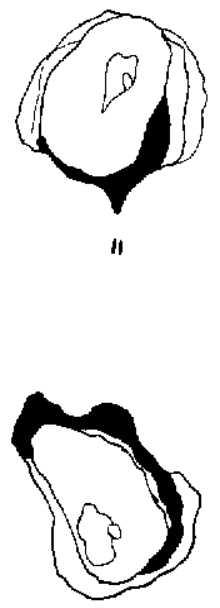

15
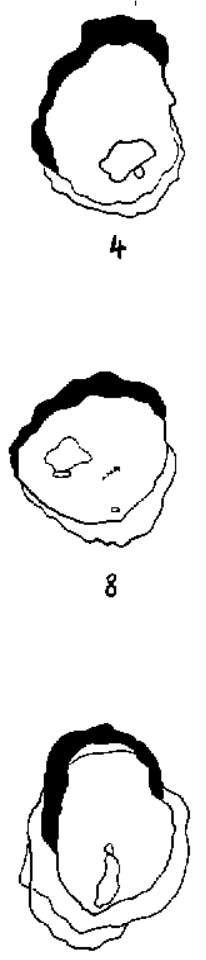

12

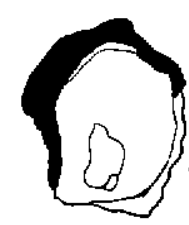

16

$10 \mu$

Planche l. - Schémas à la chambre claire de gamétocytes de L. schoutedeni 1-16 macrogaméłocyłes. 


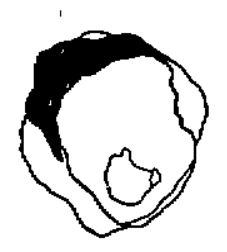

I

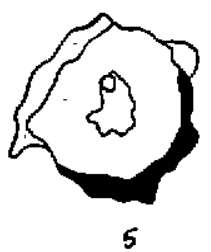

5

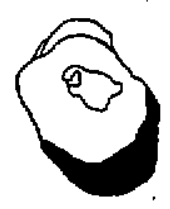

9

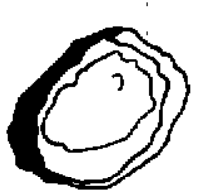

13

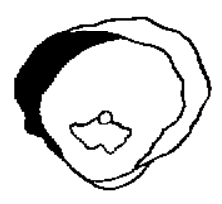

2
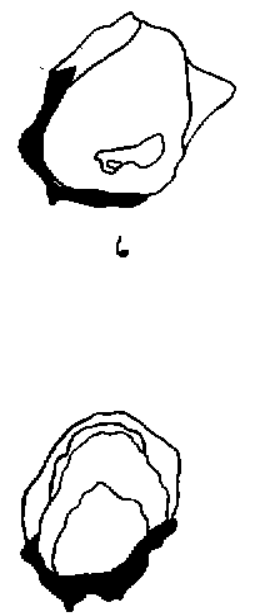

10

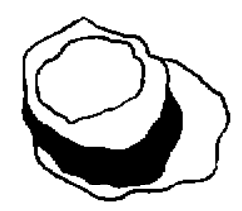

4

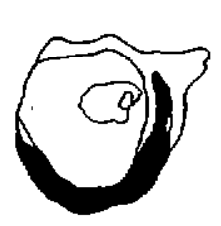

3
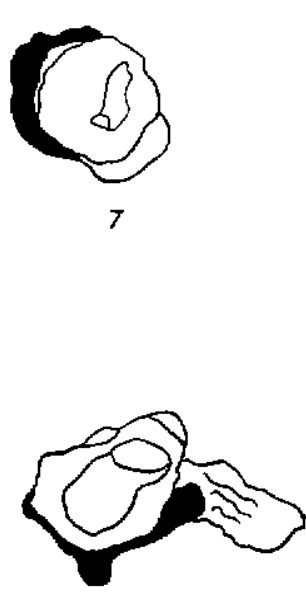

॥

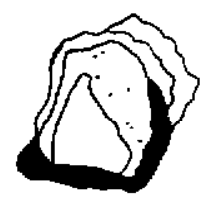

12

Planche Il. - Schémas à la chambre claire de gaméiocytes de L.schoufedeni. 1-9 macrogamétocyjes.

10-14 microgamétocytes. 
TABLFAU $\mathbb{N}^{\circ} I$

Synopsis des descriptions des Leucoytozoon sphériques de la poule domestique

\begin{tabular}{|c|c|c|c|c|}
\hline Anteur & Rodhain et al. 1913 & Atchley 1951 & Seneviratna et al.1963 & Résriltats personnels \\
\hline Locelité & Congo & U.S.A. & Ceylon & Fhodésie \\
\hline \multicolumn{5}{|c|}{ Negureg } \\
\hline Cellule hote & iusqu'à $17,5 \mu$ & $\begin{array}{l}\text { ma. } 15-17 \mu \\
\text { miz. } 13-16 \mu\end{array}$ & absent & $\begin{array}{l}\text { moyen ma. } 15,1 \mu \\
\text { moyen mi. } 14,6 \mu\end{array}$ \\
\hline Mircrogamétocyte & $12,7 \mu$ & $12-14 \gamma^{2}$ & $\begin{array}{l}5,7-14,5 \mu \\
\text { moyen } \uparrow 1,2 \mu\end{array}$ & moyen $11,8 \mu$ \\
\hline Noyau du mecrogamétocy te & 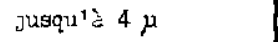 & $3-5 \mu$ & petit & moyen $4,7 \mu$ \\
\hline Macrogamétocyte & moyen $10,66 \mu$ & $10-12 \mu$ & $8-10 \mu$ & moyen $11,3 \mu$ \\
\hline Novau du tilcrogamétocyte & $7: 7 \mathrm{p}$ & - & large & moyen $9,3 \mu$ \\
\hline \multicolumn{5}{|c|}{ Descrintzon qu nacrogamétocyte } \\
\hline Granules & présent & présent souvent & absent & présent souvent \\
\hline Vacuoles & prégent & - & absent & orésent souvent \\
\hline Situation du caryosome & terminal & $\begin{array}{l}\text { haisituellernent } \\
\text { marginal }\end{array}$ & - & $\begin{array}{l}\text { terminal } 48 \mathrm{p} \cdot 100 \\
\text { marginal } 44 \mathrm{p} \cdot 100 \\
\text { intérieur } 8 \mathrm{p} \cdot 100\end{array}$ \\
\hline $\begin{array}{l}\text { Forme du noyau } \\
\qquad n\end{array}$ & ałlongé, irrégulier & $\begin{array}{l}\text { presque sphéri- } \\
\text { que parfois } \\
\text { allongé }\end{array}$ & sphérique & ellongé, irrégulier \\
\hline \multicolumn{5}{|c|}{ Description du microgamétocyte } \\
\hline Granulations & parfois présent & - & - & parfois présent \\
\hline $\begin{array}{l}\text { Relation } \\
\text { ricroganftocytes à } \\
\text { macroganétocytes }\end{array}$ & $6,6 \mathrm{p} \cdot 100$ & $\begin{array}{l}\text { beaucoup moins } \\
\text { nombreux }\end{array}$ & - & 10 p.100 \\
\hline $\begin{array}{l}\text { Noyau de la } \\
\text { celiule-h8te }\end{array}$ & présertt & $\begin{array}{l}\text { toujours } \\
\text { présent }\end{array}$ & absent & présent \\
\hline $\begin{array}{l}\text { Pourcentage de poules } \\
\text { trouvées infestées }\end{array}$ & $16,6 \div .100$ & $15,25 \mathrm{p}, 100$ & - & $59 \mathrm{p} .100$ \\
\hline Pouvoir pathogène & inepparent & inapparent & faible & inapparent \\
\hline Nom du parasite & L.schoutederi & Loandrewsi & $\underline{L_{0} \operatorname{cau} l 1 e_{x} \mathrm{i}}$ & L.schoutedeni \\
\hline
\end{tabular}


A l'exception de différences minimes dans les mesures moyennes le parasite trouvé en Rhodésie ressemble très étroitement à L. schoutedeni du Congo. ATCHLEY (1951) ne donne pas de description du noyau du microgamétocyte. Sa description ne donne pas non plus certains détails comme la présence ou l'absence de granulations chez les microgamétocytes ou de structures vacuolaires dans le cytoplasme des macrogamétoctytes. Pour le reste, sa description coincide remarquablement bien avec celle de Rodhain etal. (1913). Très probablement cette dernière description n'a pas été prise en compte par ATCHLEY (1951) à cause du fait que le nom latin de l'animal-hôte a été donné comme Gallus bankivo. G. bankiva n'existe pas en Afrique à l'état sauvage. D'autre part RODHAIN et al. (1913) nomment nettement la « poule indigène » comme hôte. Il ne peut $y$ avoir le moindre doute que ceci doit signifier la poule domestique indigène dont le nom latin accepté est Gollus domesticus.

Il est possible que le Leucocytozoon vu par COLES (1965) soit aussi L. schoutedeni, d'autant plus qu'il souligne que le parasite vu par lui est dépourvu de pouvoir pathogène.

Je n'ai pu trouver des formes en cours de développement de L. schoutedeni. Mais la similarité du parasite ressemblant à Aegypfionella vu par COLES $(1937,1939)$ avec les formes en cours de développement de L. caulleryi (Dhanapala 1962 : Seneviratna ef al., 1963) laisse supposer que c'étaient peut-être des formes en développement de L. schoutederi-L. ondrewsi.
A présent il seraił difficile de déterminer si L. schoufedeni et $L$. andrewsi sont en fait identiques. D'un côté, il y a une grande similarité sous tous les, aspects, tandis que de l'autre il y a l'important éloignement géographique des trouvailles décrites. Mais il semble qu'à cause de la parasitémie basse et de l'absence apparente de pouvoir pathogène le parasite pourrait avoir échappé à la défection dans beaucoup de cas. L'avenir pourrait révéler une présence beaucoup plus répandue de ce parasite.

\section{REMERCIEMENTS}

J'aimerais exprimer ma reconnaissance à M. T. LEES MAY, Directeur du Service vétérinaire et à M. G. J. CHRISTIE, Directeur adjoint du Service vétérinaire (Recherches) pour avoir permis l'accomplissement de ce travail, et aussi à M. W. P. BOYT, Vétérinaire en Chef (Trypanosomiase) pour avoir organisé ma visite des districts du Nord de Gokwe et de Kariba.

\section{Laboratoire de Recherches Vétérinaires, Salisbury, (Rhodésie).}

Note de l'auteur. - Après avoir envoyé cet article pour publication, j'ai trouvé que R. Rousselot (Notes de parasitologie tropicale. Paris, Vigot Frères, 1953. Tome I. p. 40) a vu chez la poule en A. O. F. un Leucocytozoon apparemment identique qu'il nomme L. gallinarum.

\section{SUMMARY}

Leucocytozoon schoutedenj has been found in several instances in the Northern region of Rhodesia in the exolic and indigenous domestic chicken, Gollus domesticus. A description of the parasite is given. The parasite is compared in detail with L. andrewsi and L. caulleryi and it is suggested that $L$. andrewsi might be identical with L. schoutedeni.

\section{RESUMEN}

Leucacylozoon schoutedeni Rhodain, Pans, Vandendranden y Bequaert, 1913, en la gallina domésfica, Gallus domesticus, en Rodesia.

En el norte de Rodesia, se encontró el Leucocytozoon schoutedeni algunas veces en la gallina doméslica exótica e indigena, Gallus domesticus. Se describe aqui el parásito. Se le compara en todas sus partes con el $L$. andrewsi y el $L$. coulleryi, y se sugiere que $L$. andrewsi y $L$. schoutedeni se parezcan. 


\section{BIBLIOGRAPHIE}

1. ATCHLEY (F.-O.). - Leucocyfozoon andrewsi $\mathrm{n}$. sp. from chickens observed in a survey of blood parasites in domestic animals in South Carolina. J. Parasit., 1951, 37, p. 483 à 488.

2. ATCHLEY (F.-O.). - Notes on Leucocytozoon andrewsi from the domestic chicken. J. Parasit., 1963, 49, p. 497 d̀ 498.

3. BERSON (J.-P.). - Les protozodires parasites des hématies et du système histiocytaire des oiseaux. Rev. Elev. Méd. Vét. Pays trop. 1964,17, p. 43 à 96.

4. COLES (J.-D.W.-A.). - A new blood parasite of the fowl. Onderstepoort. J. Vet. Sci., 1937, 9, p. 301 à 307.

5. COLES (J.-D.-W.-A.). - Aegyptianellosis of poultry. Proc. 7 th. World's Poultry Congr. 1939, Washington D.C., U. S. Dept., Agric. p. 261 d̀ 265.
6. COLES (J.-D.-W.-A.). - Communication personnelle, 1965.

7. DHANAPALA (S.-B.). - Studies on some Sporozod of Gallus domesticus and Gallus lafayetti of Ceylon. Thèse Londres, 1962.

8. GRIFFITHS (R.-B.). - Leucocyfozoon caulleryi infection. A note on recent oufbreaks in the Far East. F. A. O. Rome 06262, 1963.

9. RODHAIN (J.), PONS (C.), VANDENDRANDEN (F.) et BEQUAERT (J.). - Notes sur quelques hématozodires du Congo belge. Arch. Protistenk., 1913, 24, p. 259 d̀ 278.

10. SENEVIRATNA (P.), BANDARANAYAKE (A.) et DHANAPALA (S.-B.). - Leucocytozoon coulleryi Mathis and Leger, 1909, infection in domestic fowls in Ceylon. Ceylon Vet. J., 1963, 11, p, 2 d 8. 